Abstract 02-S5.05 Table 1 Proportion of MSM reporting self- and partner-examination of mouth, anus, penis and skin, $n=586$ )

\begin{tabular}{lllll}
\hline & & \multicolumn{3}{l}{ Frequency of examination } \\
\cline { 3 - 5 } & Body parts & Never (\%) & Once a month (\%) & At least once a week (\%) \\
\hline \multirow{2}{*}{ Self } & Mouth & 17.1 & 14.5 & 68.4 \\
& Anus & 26.4 & 24.9 & 48.7 \\
& Penis & 6.2 & 7.6 & 86.3 \\
& Skin & 8.3 & 9.7 & 81.9 \\
\multirow{3}{*}{ Partner } & Mouth & 76.6 & 7.7 & 15.6 \\
& Anus & 62.1 & 11.5 & 26.5 \\
& Penis & 47.1 & 11.6 & 41.4 \\
& Skin & 47.2 & 9.8 & 43.0 \\
\hline
\end{tabular}

Conclusions The majority of MSM reported examining themselves at least once a week, but did not examine their partners as frequently. MSM with $>3$ partners were less likely to examine their partners' bodies than those with fewer partners. Analysis of surveys from men after brochure introduction will determine whether education materials increase rates of self- and partner-examination.

\section{2-S5.06 HEALTH-SEEKING BEHAVIOURS AMONG FEMALE SEX WORKERS IN A COMMUNITY RANDOMISED TRIAL IN PERU (THE PERU-PREVEN STUDY)}

doi:10.1136/sextrans-2011-050109.96

${ }^{1} \mathrm{P}$ Kohler, ${ }^{2} \mathrm{P}$ E Campos, ${ }^{2} \mathrm{C}$ Buendia, ${ }^{2} \mathrm{C}$ Carcamo, ${ }^{2} \mathrm{P} \mathrm{J}$ Garcia, ${ }^{1} \mathrm{~J}$ Hughes, ${ }^{1} \mathrm{C}$ Mejia, ${ }^{3} \mathrm{G}$ Garnett, ${ }^{1} \mathrm{~K}$ Holmes. ${ }^{1}$ University of Washington, Seattle, USA; ${ }^{2}$ Universidad Peruana Cayetano Heredia, Lima, Peru; ${ }^{3}$ mperial College London, London, UK

Background This study aims to evaluate health-seeking and HIV/ STD preventive behaviours among FSW in mid-sized cities in Peru associated with a community randomised trial intervention and with venue of sex work.

Methods Through the Peru PREVEN multi-component intervention, mobile team outreach to FSW was conducted in an effort to lower STD rates and increase condom use as well as care-seeking from local Ministry of Health clinics for screening and evaluation of STDs. Relative risks for behavioural outcomes were calculated using multivariate Poisson regression models with robust standard errors and accounting for clustering by city. Analyses were adjusted for city-specific baseline outcomes and by brothel venue, as there were a higher proportion of brothels in intervention cities. A sub-analysis of outcomes associated with brothel venue did not control for baseline but did adjust for age, marital/cohabitation status, alcohol use, geographical region, education and randomisation arm.

Results 4156 FSW were enrolled in 20 cities; 2063 from control and 2093 from intervention cities. The median age at first paid sex was 21 years and the median duration of sex work was 20 months. Sex work was relatively frequent, with a median of 6 days worked in the last week, 4 weeks in the last month and 8 months in the last year. Frequency of sex work increased with age $(p<0.001)$. Twenty-one per cent of FSW were brothel based, 23\% street based and 56\% were bar or nightclub based. Although proportions of care-seeking behaviours were higher in intervention cities, differences were not statistically significant. In evaluating relationships of venue and health-seeking behaviours, brothel-based FSW reported significantly lower rates of non-condom use with clients $(R R=0.18 ; 95 \% \mathrm{CI}$ $0.07 \%$ to $0.44 \%$ ), and higher rates of recent health screening exams $(\mathrm{RR}=1.97$; $95 \% \mathrm{CI} 1.58 \%$ to $2.45 \%)$ and of HIV testing in the last year ( $R R=1.74 ; 95 \%$ CI $1.45 \%$ to $2.09 \%$ ), compared with FSW who were street or bar-based. Brothel-based FSW also more frequently reported knowledge of STDs ( $R R=1.07 ; 95 \%$ CI $1.04 \%$ to $1.09 \%$ ) and recognition of STD symptoms in women ( $R R=1.39 ; 95 \% \mathrm{CI}$ $1.22 \%$ to $1.59 \%)$ and in men $(R R=1.32 ; 95 \%$ CI $1.12 \%$ to $1.57 \%)$.
Conclusions Sex work venue is significantly associated with the health care-seeking and STD preventive behaviours of sex workers. Interventions to promote STD detection and prevention among FSW should consider structural or regulatory factors related to sex work venue.

\section{Social and behavioural aspects of prevention oral session 6-STI and HIV risk: geographic, demographic and behavioural heterogeneity 02-S6.01 NON-CONSENSUAL SEX AND ASSOCIATION WITH HIV INFECTION AMONG WOMEN: A COHORT STUDY IN RURAL UGANDA, 1990-2008}

doi:10.1136/sextrans-2011-050109.97

${ }^{1}$ I Birdthistle, ${ }^{2} \mathrm{~B}$ Mayanja, ${ }^{2} \mathrm{D}$ Maher, ${ }^{1} \mathrm{~S}$ Floyd, ${ }^{3} \mathrm{~J}$ Seeley, ${ }^{1} \mathrm{H}$ Weiss. ${ }^{1}$ London School of Hygiene, Tropical Medicine London, UK; ${ }^{2}$ MRC/UVRI Uganda Research Unit on AIDS, Entebbe, Uganda; ${ }^{3}$ University of East Anglia, UK

Background Non-consensual sex is associated with HIV infection in Africa, but there is little longitudinal data on this association. We describe reported non-consensual sex among women over two decades in rural southwest Uganda, including associations with incident HIV infection.

Methods Between 1990 and 2008 in rural southwestern Uganda, consenting individuals in a population cohort who recently seroconverted to HIV were enrolled into a clinical cohort, along with randomly selected HIV-negative controls. Participants were invited to the study clinic every 3 months, and females asked about recent experiences of sex against their will (since their last visit). At enrolment, associations of non-consensual sex with HIV status were analysed using conditional logistic regression. With data from all visits, this association was analysed using logistic regression, with OR adjusted for age and year of interview, allowing for within-woman correlation. Results 476 women aged 14-81 enrolled and attended 10475 visits over 19 years. At the time of enrolment, 24\% (41/188) of incident HIV and $16 \%(23 / 166)$ of HIV-negative participants reported nonconsensual sex in the past year (adjusted $\mathrm{OR}=0.93,95 \%$ CI $0.47 \%$ to $1.82 \%$ ). Among those who reported recent non-consensual sex (since their last visit) at any visit, most (80/119) did so more than once, including $48 \%$ in over half their visits. Using data from all visits, reports of recent non-consensual sex were higher among HIV-positive than HIV-negative participants (22\% vs $9 \%$; aOR $=2.29,95 \%$ $\mathrm{CI}=1.03 \%$ to $5.09 \%$ ), with the strongest associations among women aged $14-22$, those over 50 years, and married participants.

Conclusions The study shows high levels of repeated sex against one's will, with many women in this Ugandan population reporting new episodes of non-consensual sex in most or all of their visits. Nonconsensual sex was most often reported by the youngest and oldest HIV positive women. Gender-sensitive HIV programmes should address repeated sexual coercion before and subsequent to HIV infection.

\section{2-S6.02 OCCUPATIONAL AND INTIMATE PARTNER VIOLENCE AND INCONSISTENT CONDOM USE WITH CLIENTS AMONG FEMALE SEX WORKERS IN SOUTHERN INDIA}

doi:10.1136/sextrans-2011-050109.98

${ }^{1} \mathrm{~K}$ Deering, ${ }^{2} \mathrm{P}$ Bhattacharjee, ${ }^{2} \mathrm{H} \mathrm{L}$ Mohan, ${ }^{3} \mathrm{~J}$ Bradley, ${ }^{1} \mathrm{~K}$ Shannon, M C Boily, ${ }^{2} \mathrm{~B}$ M Ramesh, ${ }^{2} \mathrm{~S}$ Isac, ${ }^{5} \mathrm{~S}$ Moses, ${ }^{5} \mathrm{~J}$ Blanchard. ${ }^{1}$ University of British Columbia, Vancouver, Canada; ${ }^{2}$ Karnataka Health Promotion Trust, Bangalore, India; ${ }^{3}$ Laval University, Canada; ${ }^{4}$ Imperial College, UK; ${ }^{5}$ University of Manitoba, Winnipeg, Canada

Background Global reports suggest that interpersonal violence experienced by female sex workers (FSWs), including occupational violence (eg, by clients, CLViol) and intimate partner violence 
(IPViol) may be important structural determinants of vulnerability to HIV and sexually transmitted infections (STIs). This study characterised the type and frequency of CLViol and IPViol experienced by FSWs in southern India and examined the relationship between client violence and inconsistent condom use (ICU) with clients.

Methods Data were analysed from cross-sectional surveys of FSWs in three districts in Karnataka state (2007). ICU was defined as condom use frequency reported as 'never/sometimes/often' vs 'always', by repeat and occasional clients. CLViol and IPViol measures included having experienced physical violence in the past 6 months (ie, hurt, hit, kicked, punched, choked, burned) or sexual violence in the past year (ie, beaten or otherwise physically forced to have sexual intercourse).

Results Of our sample of 1245 FSWs, ICU was $13.0 \%$ with occasional and $20.2 \%$ with repeat clients. Overall, $13.1 \%$ of women reported physical violence from any perpetrator (CLViol: $5.5 \%$; IPViol: $4.6 \%$ ) and $9.7 \%$ reported sexual violence (CLViol: 5.2\%; IPViol: 4.4\%). In multivariable logistic regression analysis, the odds of ICU with occasional clients were significantly higher for women who had experienced physical CLViol (adjusted OR (AOR): $2.4,95 \%$ CI: $1.2 \%$ to $4.8 \%$ ) or sexual CLViol (AOR: $2.7,95 \%$ CI: $1.3 \%$ to $5.5 \%$ ). Similar results were found with repeat clients: AOR: $2.5,95 \% \mathrm{CI}: 1.4 \%$ to $4.6 \%$ and AOR $2.3,95 \%$ CI: $1.3 \%$ to $4.2 \%$, for physical and sexual violence respectively. A dose-response relationship between the number of times experiencing CLViol and increased ICU with both types of clients was also observed $(p<0.001)$ (Abstract O2-S6.02 figure 1). IPViol was not significantly associated with ICU with clients. Experiencing CLViol was not associated with experiencing IPViol $(p=0.321)$.

Discussion A strong independent relationship between experiencing client violence and inconsistent condom use with clients among FSWs in southern India was observed, highlighting FSWs' vulnerability to HIV/STI infection. Occupational violence against FSWs should be addressed within HIV/STI prevention programming. Structural-legal reforms to current sex work laws and safer-environment interventions should be developed to reduce violence and HIV/STI vulnerability among FSWs.

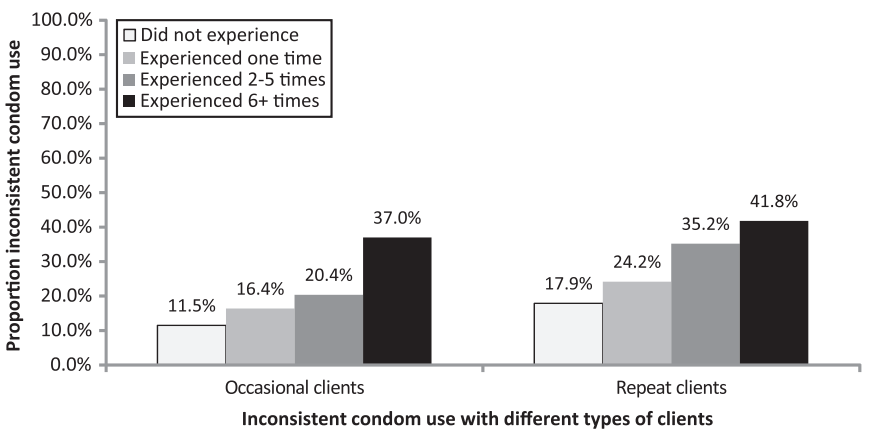

Abstract 02-S6.02 Figure 1 Proportion of female sex workers who used condoms inconsistently with occasional or repeat clients according to the number of times they experienced physical violence by clients in the last 6 months (did not experience, experienced one time, experienced 2-5 times, experienced $6+$ times).

\section{2-S6.03 HETEROGENEITY OF HIV RISK: FEMALE SEX WORKER CLIENTS AND THEIR NON-COMMERCIAL SEXUAL PARTNERS IN SOUTH INDIA}

doi:10.1136/sextrans-2011-050109.99

${ }^{1} \mathrm{~S}$ Shaw, ${ }^{2} \mathrm{~K}$ Deering, ${ }^{3} \mathrm{~S}$ Isac, ${ }^{3} \mathrm{~B}$ Ramesh, ${ }^{3} \mathrm{R}$ Washington, ${ }^{1} \mathrm{~S}$ Moses, ${ }^{1} \mathrm{~J}$ Blanchard. ${ }^{1}$ University of Manitoba, Winnipeg, Canada; ${ }^{2}$ University of British Columbia, Canada; ${ }^{3}$ Karnataka Health Promotion Trust, Bangalore, India

Introduction Clients of female sex workers (FSWs) are an important bridging population for the further transmission of HIV and other sexually transmitted infections (STIs). However, the extent of risk to non-commercial partners (NCP) of clients has rarely been quantified. This study sought to characterise the risk behaviours of clients with both non-commercial and commercial partners.

Methods Data were collected from a cross-sectional integrated behavioural and biological survey of FSW clients from five districts in Karnataka state, southern India. Clients were classified into three groups: married (and thus with an NCP); unmarried with at least one NCP; and unmarried without an NCP. Bivariate and multivariable logistic regression models were constructed to examine the association between group membership and condom use patterns with regular and casual FSWs, and where applicable, non-commercial partners. Associations between the prevalences of HIV, HSV-2, syphilis, gonorrhoeal and chlamydial infections and group membership were examined in multivariable models. Normalised weights were used to account for a complex sampling design.

Results The total sample size was 2328. Most respondents (61\%) were married, 9\% were unmarried with an NCP, and 30\% were unmarried without an NCP. Married clients were, on average 9 years older (34 vs 24.8 years), and had been visiting FSWs for an average of 12 years, vs 4 years in the other two marital categories. Compared to respondents without an NCP, married clients were at higher odds of reporting never using condoms with both casual (AOR: 1.8; 95\% CI: $1.3 \%$ to $2.4 \%, p<0.0001$ ) and regular (AOR:1.8; $95 \%$ CI $1.2 \%$ to $2.7 \%, p=0.009)$ FSWs. Among the two groups reporting an NCP, $91 \%$ of married respondents reported never using condoms with their NCP, compared to $62 \%$ of unmarried clients (AOR: $5.2 ; 95 \% \mathrm{CI}$ $3.3 \%$ to $8.1 \%, p<0.0001)$. HIV prevalence was $6 \%, 5 \%$ and $8 \%$ among those that were married, those unmarried without an NCP, and those unmarried with an NCP, respectively $(p=0.384)$. For the same groups, HSV-2 prevalence was $37 \%, 16 \%$ and $19 \%(p<0.0001)$. In adjusted analyses, married respondents remained at highest odds of being infected with HSV-2 (AOR: 1.5 ; $95 \%$ CI $1.1 \%$ to $2.1 \%$, $\mathrm{p}=0.006$ ).

Conclusion Married respondents were least likely to use condoms with both commercial and non-commercial sexual partners, while also having the highest prevalence of HSV-2. These findings illustrate the high risk posed to both commercial and non-commercial partners of married clients of FSWs.

\section{2-S6.04 AN EXPLORATORY SURVEY OF MALE SEX WORKERS AND HIV RISK IN AN URBAN AREA OF SOUTHWEST CHINA}

doi:10.1136/sextrans-2011-050109.100

${ }^{1} \mathrm{~B}$ N Yu, ${ }^{2} \mathrm{X}$ A Wang, ${ }^{2} \mathrm{~F}$ Yu, ${ }^{3} \mathrm{~J}$ Wang, ${ }^{1} \mathrm{~J}$ Blanchard. ${ }^{1}$ University of Manitoba, Winnipeg, Canada; ${ }^{2}$ Chengdu Gay Care Organization, Chengdu, China; ${ }^{3}$ China Male Tongzhi Health Forum, Chengdu, China

Background Little knowledge is available on risk behaviours of HIV and basic demographic characteristics in male sex workers (MSW) in the population of men who have sex with men (MSM) in China. The objective of this study is to explore the risk to HIV/STI of MSW in southwest China through a convenience sample of an HIV surveillance survey of MSM

Method Those who self-reported had been paid for sex or "engaging in money exchange for sex" were identified as MSW or "Money Boys". Data of the past five cycles (2004-2008) of an HIV surveillance survey were included in this study. Descriptive statistics were employed to describe demographics, sex practices, and HIV prevalence in these male sex workers.

Results A total of 117 men self-identified as MSW in five consecutive cross-sectional surveys (2004 n=54, $2005 \mathrm{n}=39,2006 \mathrm{n}=45,2007$ $\mathrm{n}=34$, and $2008 \mathrm{n}=45$ ). The median age of participants ranged from 25 to 27 years. In the 6 months prior to the survey, the median number of male sex partners ranged from 4 to 11; the median of 